\title{
CAPÍTULOIV
}

\section{EFECTOS DEL CONTROL FISCAL EN EL APRENDIZAJE ORGANIZACIONAL DE LAS CONTRALORÍAS}

\section{Effects of fiscal control on the organizational learning of comptrollers}

Jhonny Marlon Cárdenas Arévalo58.

\section{Resumen:}

El impacto del control fiscal se debe ver reflejado en la gestión de los sujetos de control, específicamente en la operación de sus procesos. Esta investigación tiene como propósito validar tal hipótesis y para ello se ha valido de una herramienta que condensa los resultados de las auditorías, anualmente practicadas a las contralorías de Colombia, por la Auditoría General de la República, el plan de mejoramiento. Se analizaron los últimos siete planes de mejoramiento de tres contralorías, entre ellas la Contraloría General de la República y se compararon sus hallazgos con el ánimo de verificar si estos se repetían año a año. De esta manera se estableció la curva de aprendizaje de cada sujeto de control respecto de sus procesos operativos.

\section{Abstract:}

The impact of fiscal control must be reflected in the management of control subjects, specifically in the operation of their processes. The purpose of this investigation is to validate

58 Ingeniero Industrial de la Universidad Distrital Francisco José de Caldas. Psicólogo de la Universidad Nacional de Colombia. Especialista en Ingeniería de Calidad y el Comportamiento de la Universidad Católica de Colombia. Magíster en Estudios y Gestión del Desarrollo de la Universidad de la Salle. Asesor de Gestión del Auditor General de la República. Investigador "Observatorio de Política Pública del Control Fiscal" adscrito -según convenio- a la Auditoría General de la República y la Universidad Santo Tomás de Bogotá.

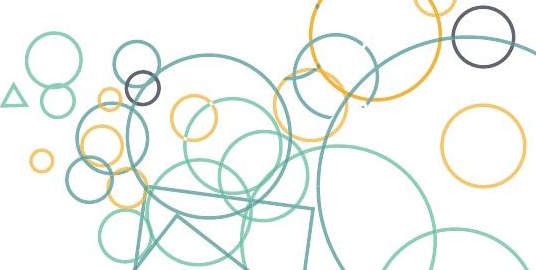


this hypothesis and for this purpose it has used a tool that condenses the results of the audits, annually carried out to the comptrollers of Colombia, by the General Audit of the Republic, the improvement plan. The last seven improvement plans of three comptrollerships were analyzed, among them the Comptroller General of the Republic and their findings were compared in order to verify whether they were repeated year after year. In this way, the learning curve of each control subject with respect to its operational processes was established.

\section{Palabras clave:}

Aprendizaje organizacional; plan de mejoramiento; proceso auditor; control fiscal; curva de aprendizaje.

\section{Key words:}

Organizational learning; Improvement plan; audit process; fiscal control; learning curve.

Temario: 4.1. Marco conceptual. 4.2. Pretensión. 4.3. Procedimiento Empleado. 4.4. Resultados. 4.5. Conclusiones. 4.6. Bibliografía.

\section{Introducción}

El control fiscal es una función pública orientada a verificar la correcta destinación y uso de los recursos públicos de un país. Tal función es desarrollada por entidades del Estado, cuya estructura, organización y funcionamiento son similares a las de las empresas del sector privado. En ese sentido, el comportamiento de las organizaciones del sector privado y del sector público puede ser analizado utilizando las mismas herramientas. Dentro del amplio panorama de fenómenos que comprende el comportamiento organizacional, está el aprendizaje. Esta capacidad institucional se puede reflejar en diferentes escenarios, de acuerdo con la naturaleza de cada organización. Tal es el caso de aquellas entidades dedicadas a ejercer la vigilancia del erario. Estas, al igual que sus sujetos vigilados deben ser eficaces, eficientes y efectivas en su actuar y para ello deben tener en cuenta sus yerros y a partir de los mismos aplicar correctivos adecuados, de tal suerte que no se vuelvan a repetir, es decir, las entidades deben aprender de sus errores. Este documento, a partir del análisis del plan de mejoramiento de las contralorías territoriales, interpreta la manera en que se desarrolla el aprendizaje organizacional.

\subsection{MARCO CONCEPTUAL}

\subsubsection{Objeto del control}

De acuerdo con el artículo 1 de la Ley 87 de 1993 por la cual se establecen normas para el ejercicio del control interno en las entidades y organismos del Estado, el control interno 
se define como: “(...) sistema integrado por el esquema de organización y el conjunto de los planes, métodos, principios, normas, procedimientos y mecanismos de verificación y evaluación adoptados por una entidad. (...)"y su propósito es:

(...) procurar que todas las actividades, operaciones y actuaciones, así como la administración de la información y los recursos, se realicen de acuerdo con las normas constitucionales y legales vigentes dentro de las políticas trazadas por la dirección y en atención a las metas u objetivos previstos. (p.1).

Desde una perspectiva administrativa, Ayala (2007) citando a Berry (2005) señala que el control se identifica como el:

(...) proceso de conducir a las organizaciones hacia patrones de actividad viables en un entorno cambiante", lo cual implica que el control se refiere a la acción de influir "sobre el comportamiento de (...) los miembros de la organización de forma que se consigan al menos, algunos de los objetivos organizacionales.

Atendiendo las diferentes dimensiones en las que se presenta el control, bien sea como un conjunto de mecanismos, como un proceso, como una capacidad o como un medio, Ayala (2007) propone la siguiente definición integrada:

El control organizacional es aquel proceso que, basándose en la capacidad de influencia sobre las personas, trata de asegurar que el desempeño de los individuos se oriente a los objetivos fijados por la organización, aplicando las medidas correctoras necesarias en cada caso.

En síntesis, el control es una función inherente a la administración de una organización, que se realiza como un proceso, se soporta en instrumentos metodológicos y busca el cumplimiento de los planes institucionales que lo enmarcan.

\subsubsection{Aprendizaje organizacional}

Si el control es una función organizacional para asegurar el cumplimiento de los planes institucionales, entonces, es pertinente plantear las definiciones que existen de aprendizaje organizacional.

En palabras de Castañeda, para Fasterby-Smith y Lyles (2003), “(...) el elemento característico del aprendizaje organizacional es el proceso mediante el cual las entidades, a partir de individuos, adquieren, construyen y transfieren conocimiento."

El aprender se puede entender como una capacidad y en el caso de las organizaciones, Garzón (2012) afirma que esta capacidad se define como:

(...) el potencial dinámico de creación, asimilación, difusión y utilización del conocimiento por medio de numerosos flujos de conocimiento que hacen posible la formación y evolución de las memorias organizacionales de conocimiento que capacitan a las organizaciones y sus agentes de conocimiento para actuar intencionalmente en entornos cambiantes (Prieto, 2004).

En conclusión, el aprendizaje organizacional se ve favorecido por la información que genera el 


\section{OPPCF}

proceso auditor, es decir por la aplicación del control. Uno de los productos facticos del control realizado a través del proceso auditor, es el plan de mejoramiento, el cual contiene aquellas actividades que presentan errores o fallas en su ejecución y que como tal, deben ser tratadas para asegurar que dicha situación no vuelva a presentarse.

\subsubsection{El plan de mejoramiento, instrumento para el aprendizaje}

Conforme el Manual del Proceso Auditor, versión 9.1 publicado por la Auditoría General de la República en 2018:

"El plan de mejoramiento es el documento que contiene el conjunto de acciones que ha decidido adelantar un sujeto de control fiscal, tendientes a subsanar o corregir los hallazgos identificados en ejercicio de la auditoría (...), (...) con el fin de adecuar la gestión fiscal a los principios de economía, eficiencia, eficacia, equidad o a mitigar el impacto ambiental. Sobre dicho plan, la AGR no emitirá pronunciamiento sobre las acciones propuestas; dentro del siguiente proceso auditor, se evaluará la efectividad de las mismas para eliminar las causas que originaron los hallazgos." (p. 32)

Como se aprecia el plan de mejoramiento permite ver la falla (hallazgo) y sus causas, y con base en las mismas, el sujeto de control formular acciones para superar esta situación (acciones de mejora). Se espera que, de ser efectivo el diseño de las acciones de mejora, las causas de eliminen y el hallazgo no se vuelva a presentar.

\subsubsection{La curva de aprendizaje}

Este concepto fue desarrollado por Theodore P. Wright en 1936 en el marco de la industria aeronáutica y lo empleo para explicar la relación entre la cantidad de producción alcanzada y el tiempo necesario para ello. Al repetir el ejercicio, la producción debe aumentar en el mismo periodo de tiempo o menos.

Para el propósito de este estudio, el concepto de curva de aprendizaje permite ver la relación entre los hallazgos o presencia de errores en la gestión de una contraloría a lo largo del tiempo. Cada vez que se realiza una auditoría a una contraloría, los hallazgos o errores en un proceso específico, deben disminuir. Esta es la curva de aprendizaje de la contraloría a partir del control fiscal.

\subsection{PRETENSIÓN}

El objetivo de esta investigación se fundamenta en los beneficios organizacionales que trae el ejercicio del proceso auditor para los sujetos de control. En el entendido de que este último es el medio a través del cual una organización, en este caso una contraloría, identifica los aspectos 
operativos en los que está fallando y sobre ellos implementa acciones de mejora.

En definitiva la pretensión es demostrar que: "el proceso auditor, a través del plan de mejoramiento, genera aprendizaje organizacional en las contralorías".

Desde un punto de vista contrario, es decir, utilizando una premisa que permita falsear la pretensión, es posible afirmar que "la recurrencia de un mismo hallazgo en dos o más planes de mejoramiento de un mismo sujeto de control, demuestra la inefectividad del proceso auditor."

De lo anterior se desprende que es una situación indeseable que un hallazgo se repita en dos o más planes de mejoramiento. De allí que una organización que efectivamente aprende no repite hallazgos en sus planes de mejoramiento, toda vez que ha transformado aquellas actividades identificadas en el mencionado plan, producto del proceso auditor.

\subsection{PROCEDIMIENTO EMPLEADO}

\subsubsection{Muestra - Criterios}

Para el desarrollo de esta investigación se utilizaron, como fuente de información, los planes de mejoramiento de las contralorías.

En términos de tiempo, fueron analizados los planes de mejoramiento de las vigencias 2012 a 2018.

Para un total de 455 planes de mejoramiento presentados en el periodo de tiempo señalado, se diseñó una muestra que permitiera la factibilidad del análisis. El criterio para seleccionar los planes de mejoramiento fue: Por cada vigencia se seleccionaron las contralorías con mayor y menor presupuesto de funcionamiento. Esto como una manera de desvirtuar la posible asociación entre capacidad presupuestal y aprendizaje.

\subsubsection{Categorías descriptivas}

Para el procesamiento de la información se diseñó una matriz que permitió comparar los planes de mejoramiento de los siete años de análisis. De cada plan de mejoramiento se extrajeron las columnas "causa", "efecto" y "condición", las cuales constituyen elementos que caracterizan el hallazgo.

Para facilitar la identificación de patrones, tendencias o similitudes entre hallazgos, estos se asociaron a categorías genéricas de información como los procesos a través de los cuales opera un sujeto de control. En suma, cada hallazgo fue asociado a un proceso, que en la matriz hizo posible identificar el proceso sobre el que debía aprender el sujeto de control.

Debe interpretarse entonces que, cada categoría encontrada es un proceso de gestión sobre

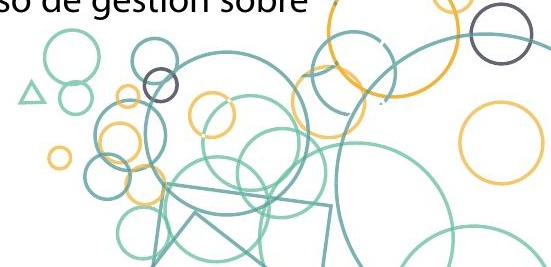


el cual debe mejorar su aprendizaje la contraloría.

\subsection{RESULTADOS}

Dado que el presupuesto asignado al funcionamiento de las contralorías, varía cada año, las parejas establecidas no fueron las mismas para cada vigencia. Por lo tanto, se realizó un análisis efectivo a los siete planes de mejoramiento de tres contralorías: Contraloría General de la República, Contraloría de Medellín y Contraloría del Vaupés.

La cantidad esperada de hallazgos por proceso, anualmente es cero. Si un hallazgo no se repite en el siguiente año, demuestra que la organización aprendió sobre dicho proceso y que el control le permitió identificar aquellos procesos en los que debía aprender.

Entonces, si al graficar el número de hallazgos sobre un mismo proceso a lo largo de ocho años, la curva de aprendizaje tiende a cero, se entiende que hay un desarrollo progresivo de la entidad hacia el aprendizaje en esa materia.

\subsubsection{Análisis por categoría descriptiva}

A continuación se presenta la frecuencia con la que se identificaron hallazgos en los planes de mejoramiento. 
Tabla 1. Frecuencia de los hallazgos, distribuida por proceso y contraloría en el periodo $2012-2018$

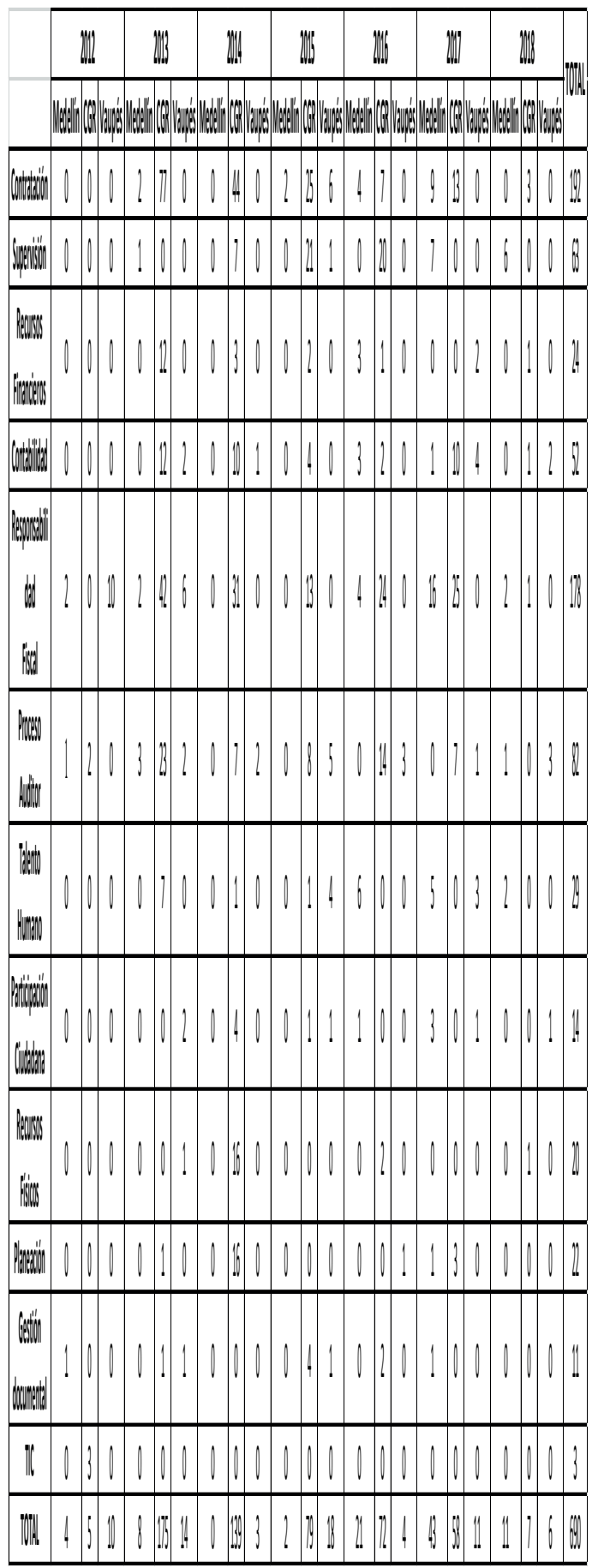




\subsubsection{Categorías - Procesos:}

Las categorías identificadas en las bases de datos analizadas fueron: Contratación; Supervisión; Financiera; Contabilidad; Responsabilidad Fiscal; Proceso Auditor; Talento Humano; Participación Ciudadana; Recursos Físicos; Planeación; Gestión documental y TIC.

\subsubsection{Curvas de aprendizaje}

A continuación se presentan los resultados obtenidos frente al aprendizaje por proceso. Estos valores son acumulados entre los tres sujetos de control.

\section{Gráfico 1. Curva de aprendizaje de Contratación}

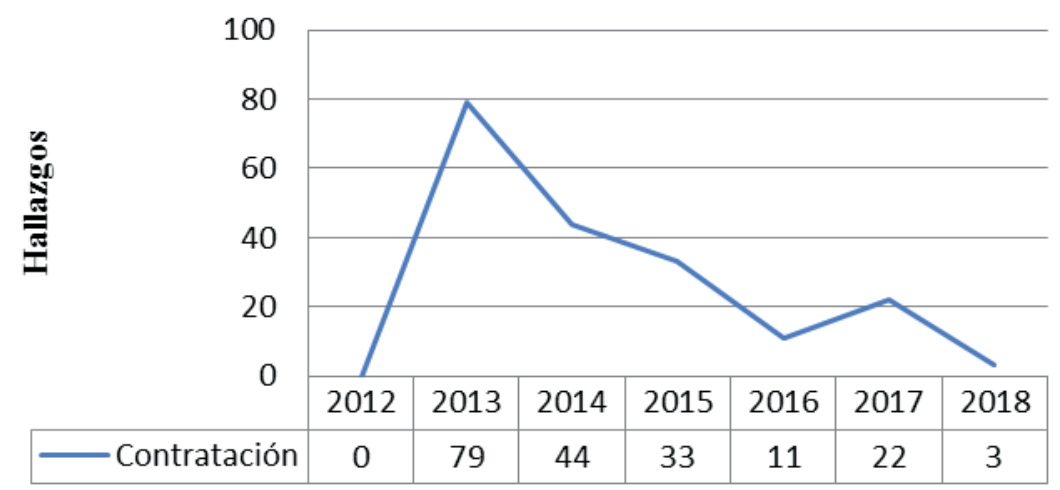

Fuente: Elaboración propia

Gráfico 2. Curva de aprendizaje de Supervisión

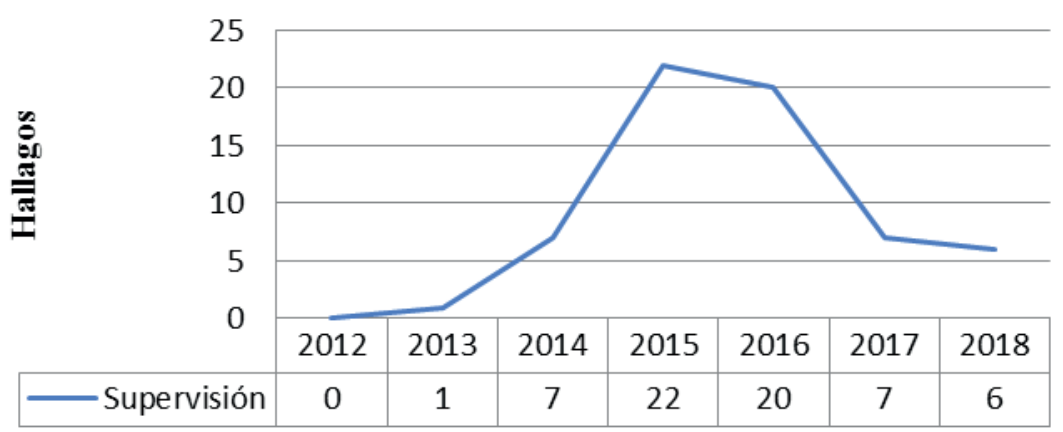

Fuente: Elaboración propia 
Gráfico 3. Curva de aprendizaje de Recursos Financieros

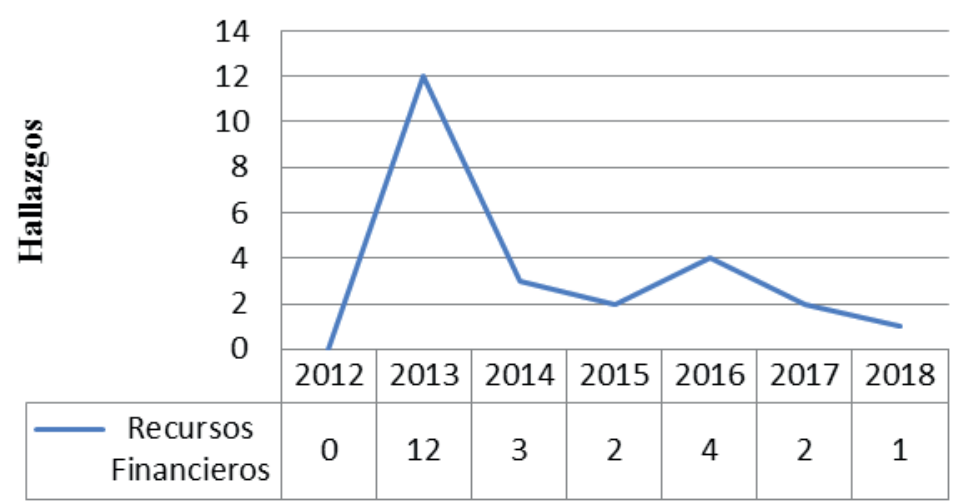

Fuente: Elaboración propia

\section{Gráfico 4. Curva de aprendizaje de} Contabilidad

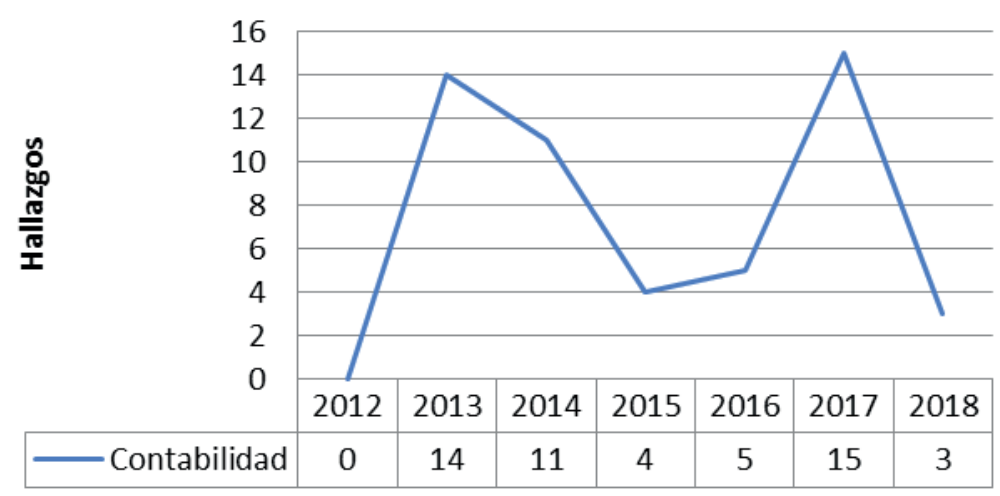

Fuente: Elaboración propia 
Gráfico 5. Curva de aprendizaje de Responsabilidad

Fiscal

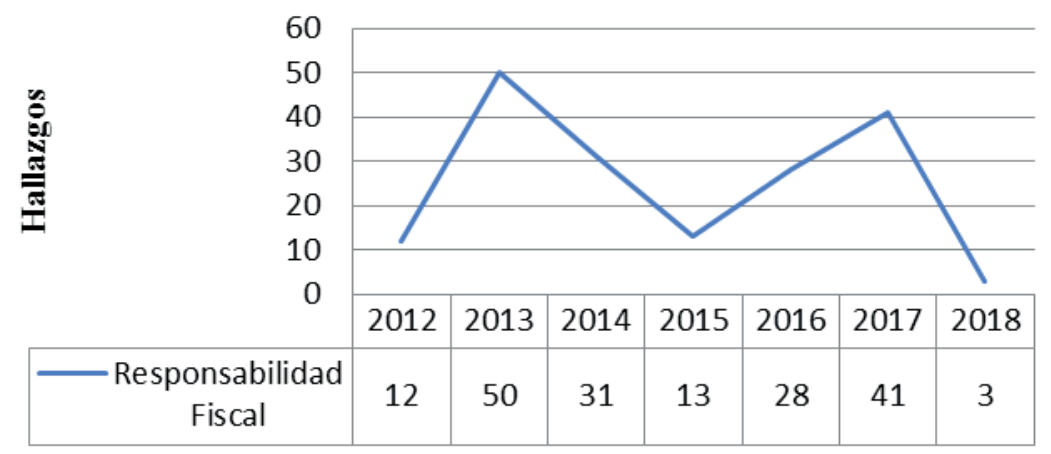

Fuente: Elaboración propia

Gráfico 6. Curva de aprendizaje de Proceso Auditor

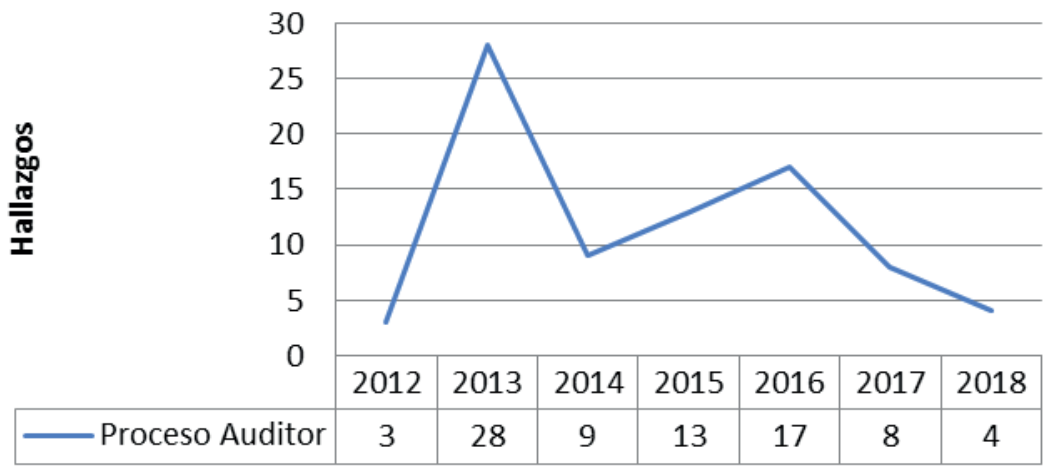

Fuente: Elaboración propia

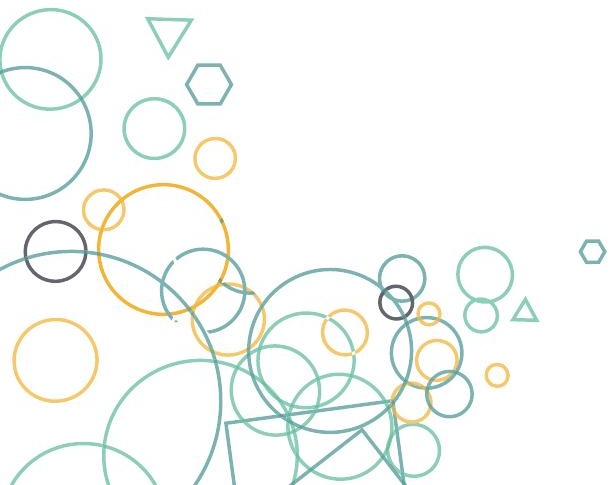


Gráfico 7. Curva de aprendizaje de Talento

\section{Humano}

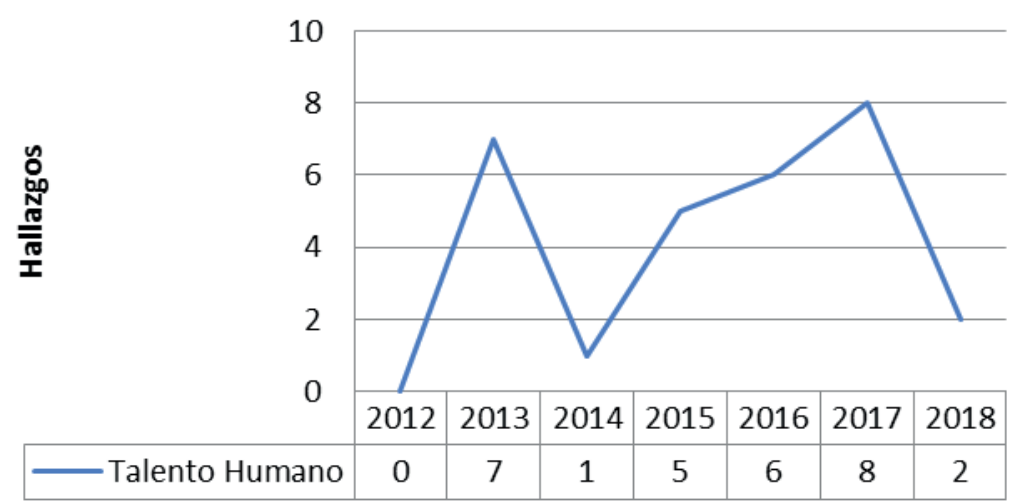

Fuente: Elaboración propia

\section{Gráfico 8. Curva de aprendizaje de}

\section{Participación Ciudadana}

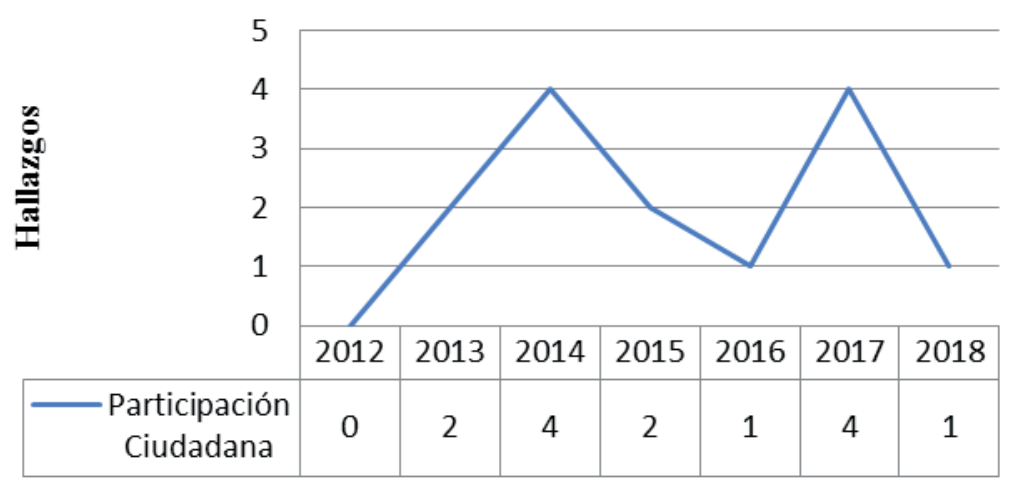

Fuente: Elaboración propia 
Gráfico 9. Curva de aprendizaje de Recursos Físicos

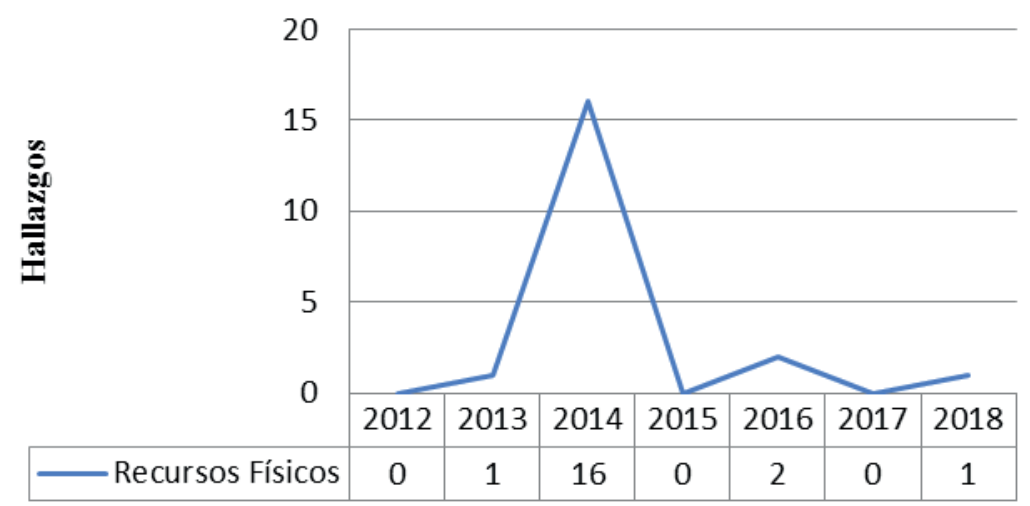

Fuente: Elaboración propia

Gráfico 10. Curva de aprendizaje de Planeación

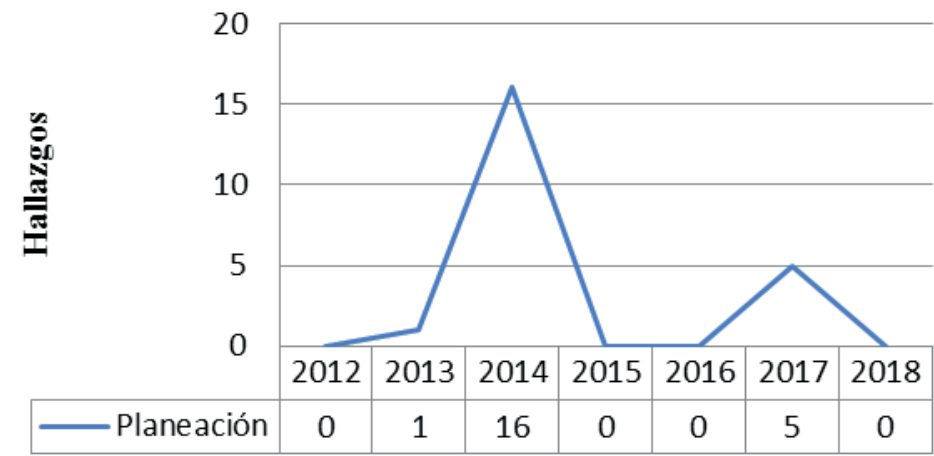

Fuente: Elaboración propia

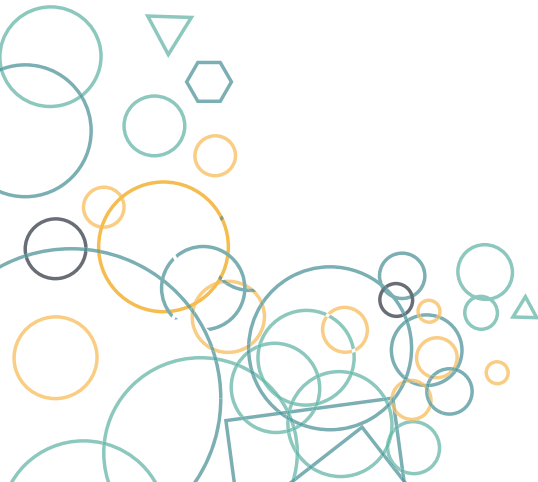


Gráfico 11. Curva de aprendizaje de Gestión documental

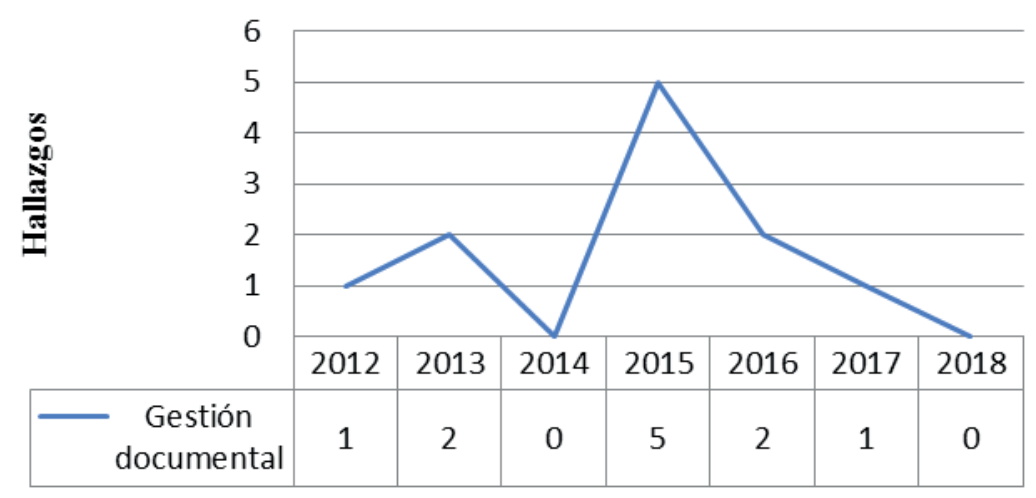

Fuente: Elaboración propia

\subsubsection{Análisis por contraloría}

A continuación se presentan, por contraloría, solo aquellos procesos que evidencian aprendizaje en los últimos siete años.

Gráfico 12. Curva de aprendizaje en Contratación CGR

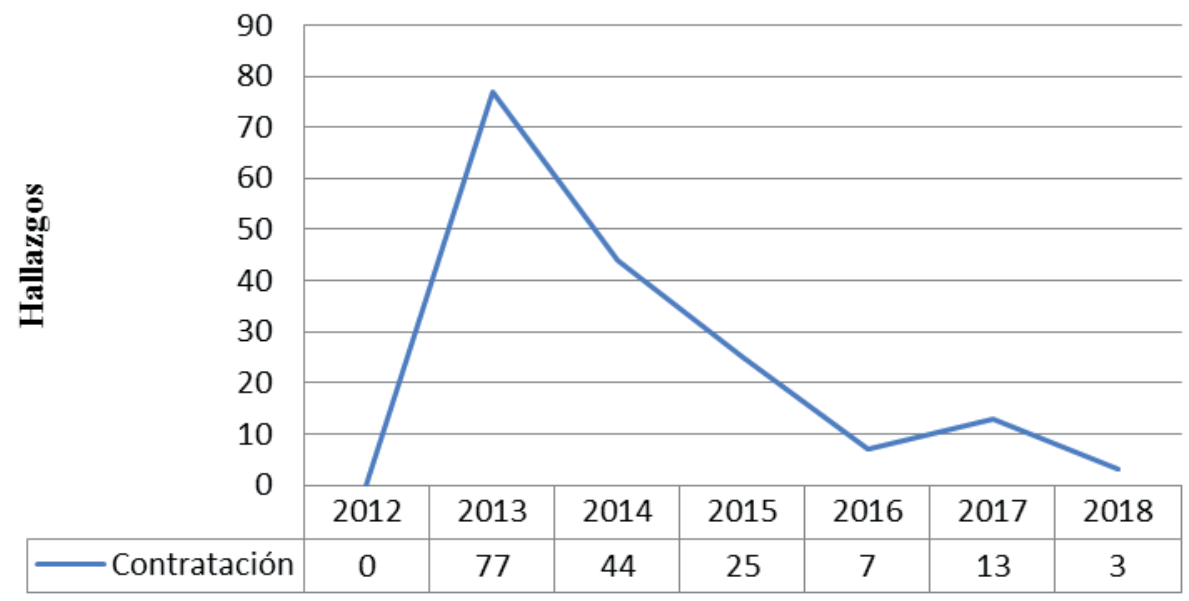

Fuente: Elaboración propia 
Gráfico 13. Curva de aprendizaje en Supervisión CGR

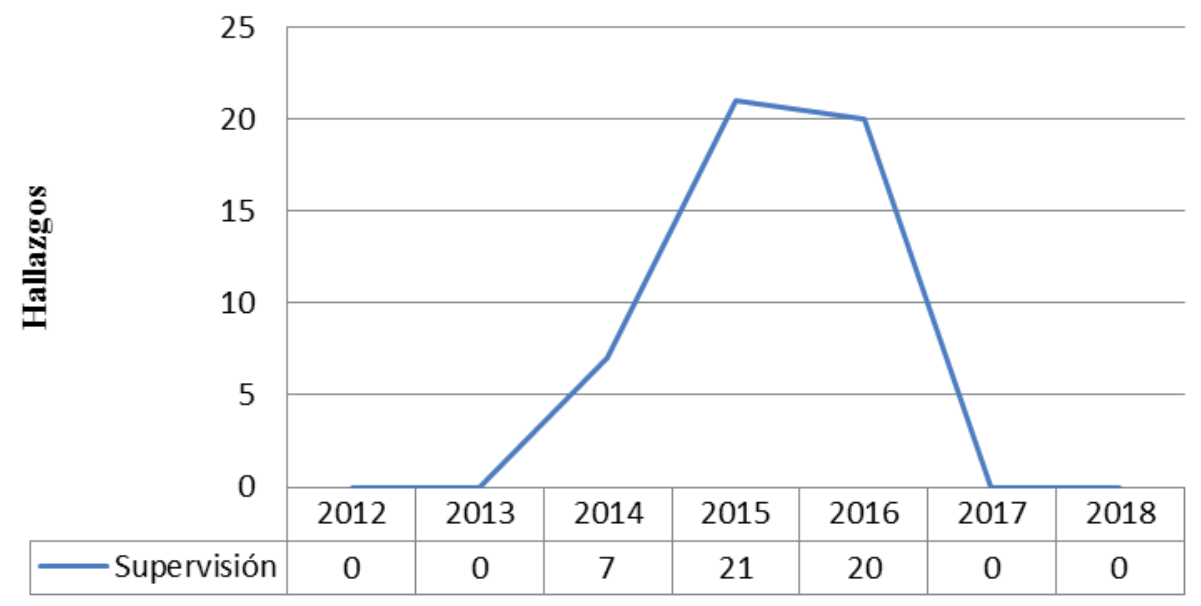

Fuente: Elaboración propia

En supervisión de contratos la contraloría mejoró su desempeño en los últimos tres años.

Gráfico 14. Curva de aprendizaje en Recursos

Financieros

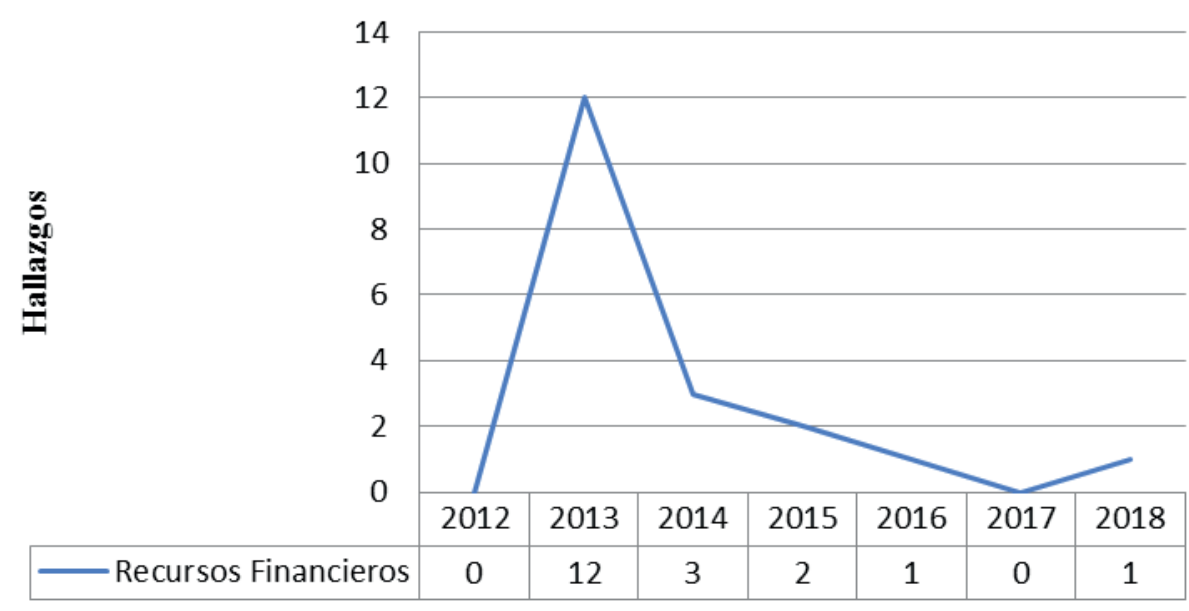

Fuente: Elaboración propia 


\section{Gráfico 15. Curva de aprendizaje en Contabilidad -}

Contraloría de Medellín

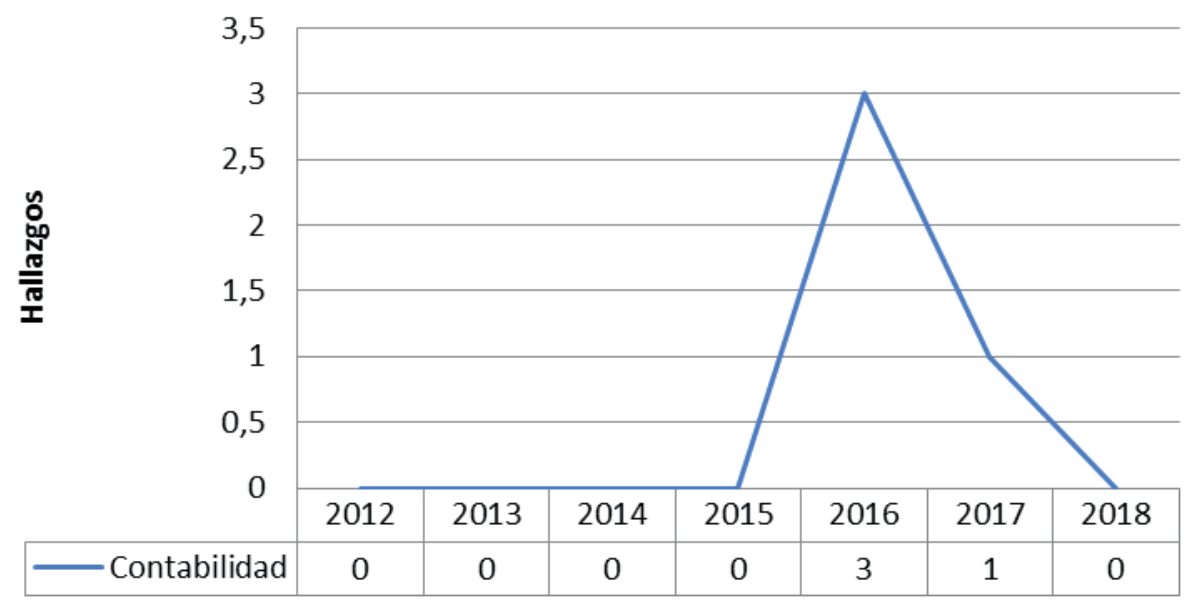

Fuente: Elaboración propia

A partir de las fallas detectadas en 2016, la contraloría desarrolló el aprendizaje suficiente para disminuir y eliminar las causas de los hallazgos.

Gráfico 16. Curva de aprendizaje en Responsabilidad Fiscal - Contraloría del Vaupés

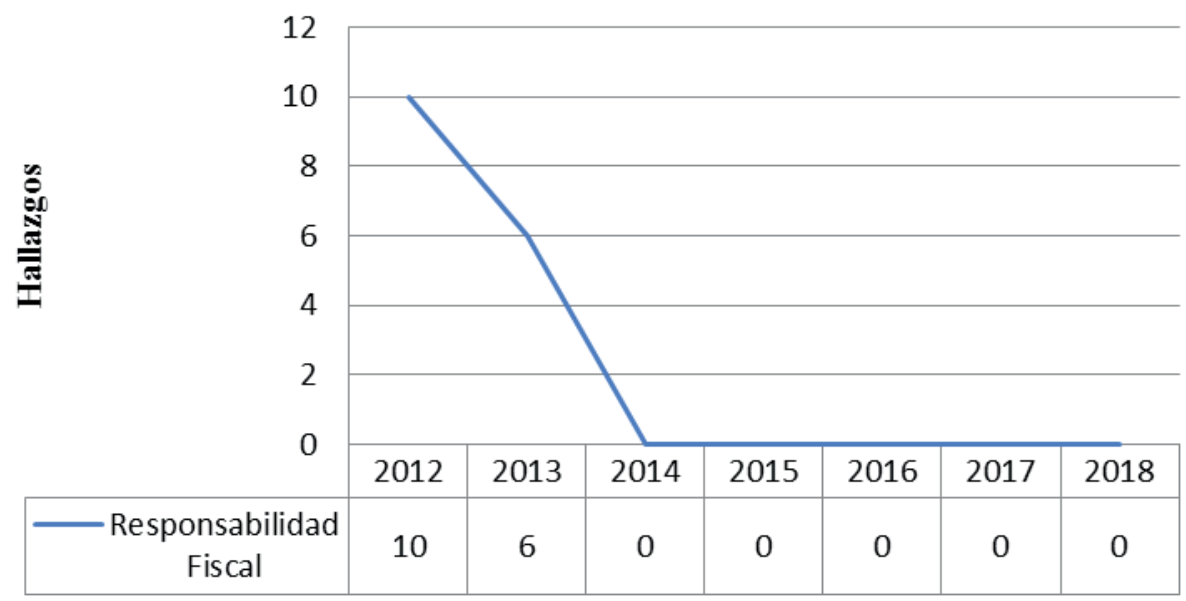

Fuente: Elaboración propia 
La Contraloría de Vaupés, en materia del proceso de responsabilidad fiscal, demuestra la sostenibilidad de su aprendizaje, al no presentar hallazgos en esta materia durante los últimos cinco años.

Gráfico 17. Curva de aprendizaje en Proceso Auditor CGR

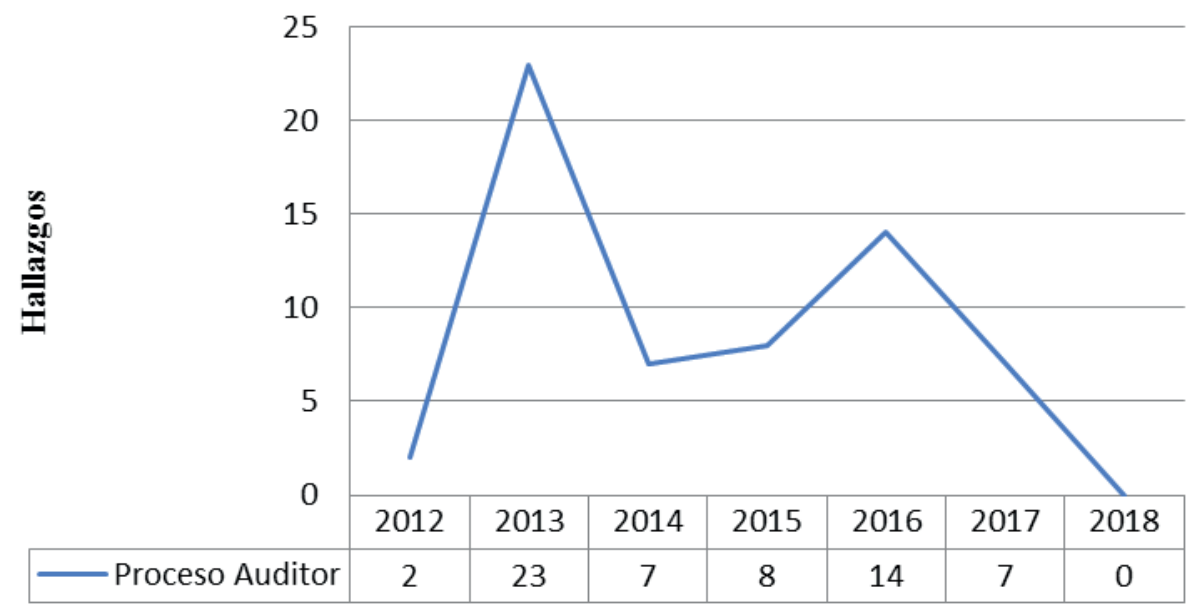

Fuente: Elaboración propia

Gráfico 18. Curva de aprendizaje en Talento Humano CGR

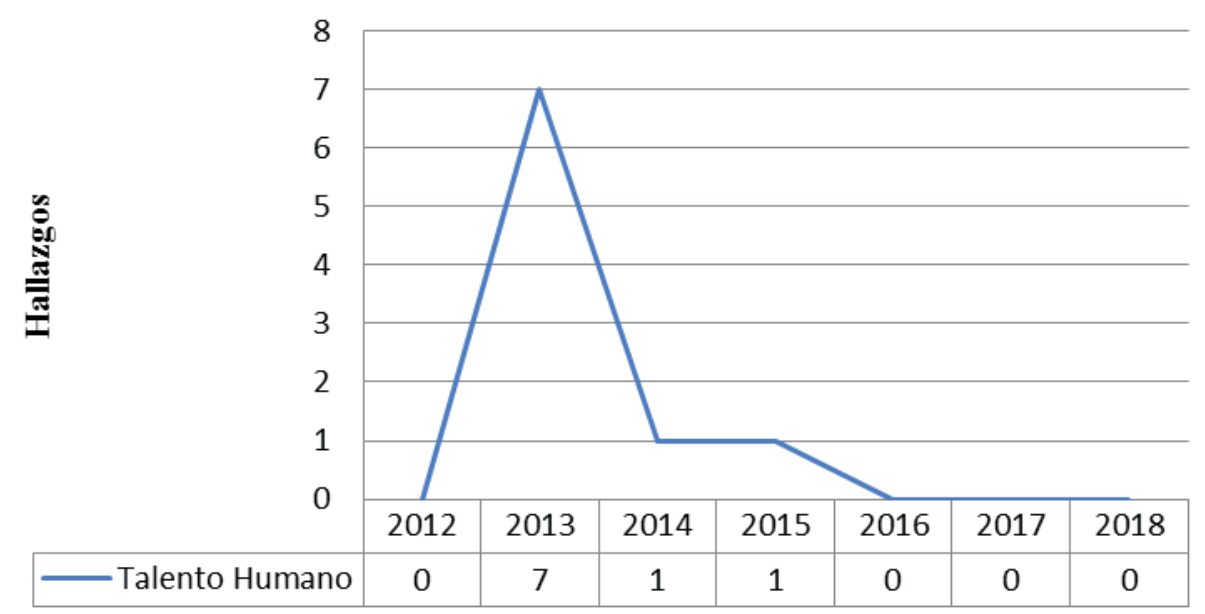

Fuente: Elaboración propia 


\section{Gráfico 19. Curva de aprendizaje en Talento Humano} Contraloría del Vaupés

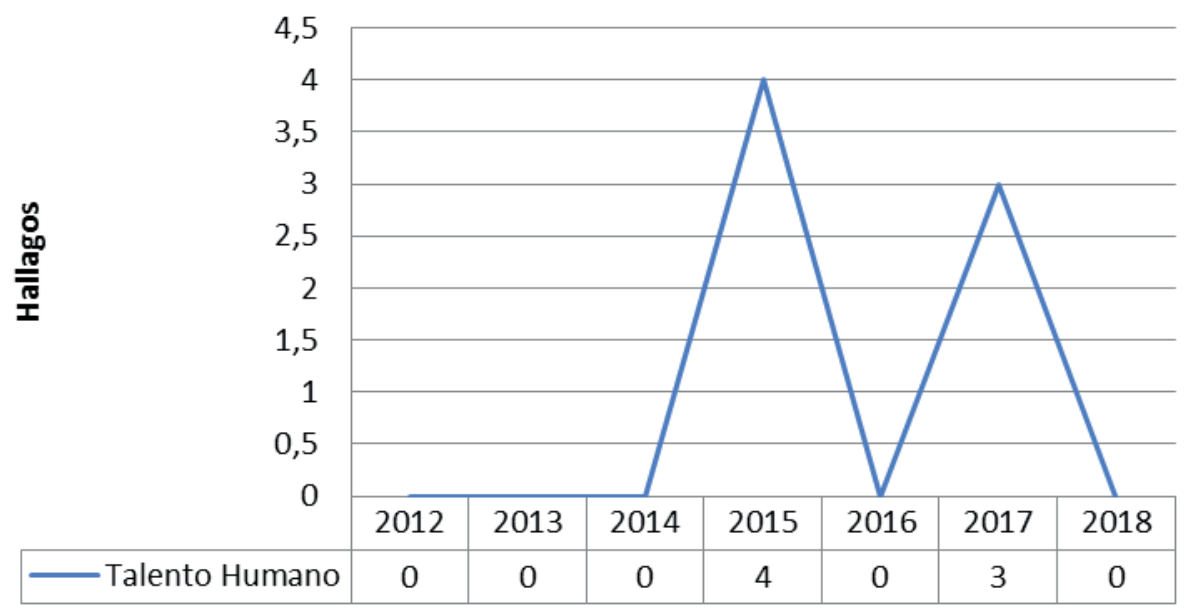

Fuente: Elaboración propia

En el caso del proceso de gestión del talento humano que administra la Contraloría del Vaupés, es evidente que su aprendizaje no se ha dado en la medida en que a pesar de haber reducido a cero los hallazgos de un año a otro, en el siguiente estas situaciones volvieron a presentarse.

Gráfico 20. Curva de aprendizaje en Participación Ciudadana - CGR

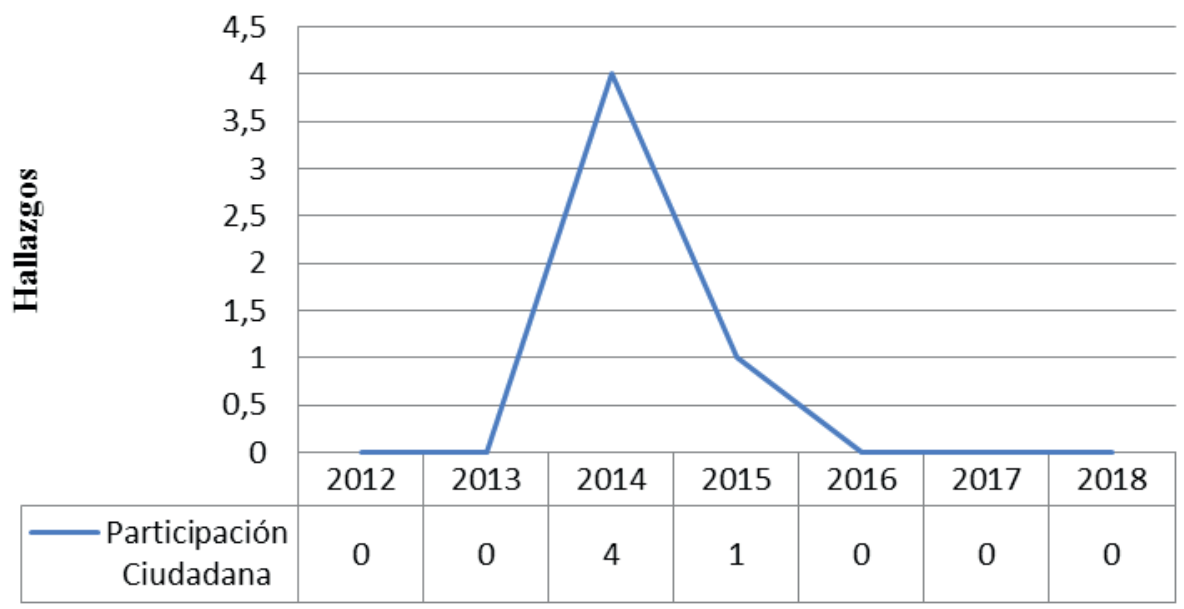

Fuente: Elaboración propia

En la Contraloría General de la República se evidencia que en materia del proceso de participación ciudadana, las acciones de mejora implementadas desde 2014 han generado el 


\section{OPPCF}

aprendizaje necesario para realizar el proceso cumpliendo los parámetros previstos, y por ello no se presentan hallazgos desde hace tres años.

\subsection{CONCLUSIONES}

Los resultados agrupados por proceso demuestran que gracias a los hallazgos encontrados, en el marco del control fiscal, los sujetos vigilados desarrollan acciones de mejora (aprenden), que les permite superar la situaciones encontradas. En este sentido, se cumple la hipótesis: el proceso auditor genera aprendizaje organizacional en los sujetos de control.

Por contraloría, los resultados demuestran que no en todos los procesos el aprendizaje es sostenible. Es decir, una contraloría puede volver a repetir hallazgos sobre un mismo proceso después de dos años.

En materia de contratación y supervisión de contratos es en donde más, las instituciones han aprendido. Esto obedece a que es el proceso que más hallazgos presentó hacia el año 2012 y los cuales se han visto disminuidos paulatinamente.

El proceso auditor, eje de la misión de las contralorías, demuestra un aprendizaje que se ha sostenido con el tiempo, pues los hallazgos se han disminuido en cada vigencia.

El proceso misional que no demuestra aprendizaje es el de responsabilidad fiscal. Lo anterior se sustenta que en entre 2012 y 2018 se siguen presentando el mismo número de hallazgos y las acciones de mejora aplicadas no se han mantenido en el tiempo.

Es necesario hacer este análisis comparativo para cada una de las contralorías pues estos resultados sirven de insumo para retroalimentar el proceso auditor que realiza la Auditoría General de la República. Al elaborar el plan general de auditorías, saber el estado del aprendizaje por procesos, ayuda a focalizar la muestra de auditoría, es decir, a priorizar la vigilancia de aquello que no ha mejorado en el sujeto de control.

Es pertinente profundizar este tipo de análisis para identificar los factores que impiden la sostenibilidad del aprendizaje organizacional.

\subsection{BIBLIOGRAFÍA}

Auditoría General de la República. (29 de Enero de 2019). auditoria.gov.co. Recuperado el 02 de noviembre de 2018, de http://www.auditoria.gov.co/

Ayala Calvo, J. C. (2007). Dialnet. Recuperado el 16 de julio de 2019, de https://dialnet.unirioja. es 
Castañeda, D. \&. (2007). Dialnet. Recuperado el 15 de junio de 2019, de https://dialnet.unirioja. es

Congreso de la República de Colombia. . (1993). funcionpublica.gov.co. Recuperado el 17 de julio de 2019, de https://www.funcionpublica.gov.co/eva/gestornormativo/norma. php?i=300

Garzón Castrillón, M. A. (2008). Dialnet. Recuperado el 15 de octubre de 2018, de https:// dialnet.unirioja.es

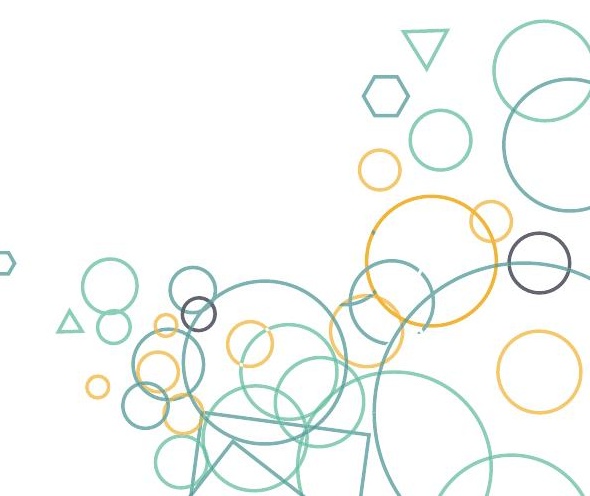

